\title{
Bone morphogenetic protein 2 regulates the differentiation of nitrergic enteric neurons by modulating Smad1 signaling in slow transit constipation
}

\author{
XULIANG LIU ${ }^{1 *}$, SHANGMING LIU ${ }^{2 *}$, YANAN XU ${ }^{3}$, XIUQIN LIU $^{4}$ and DAQING SUN ${ }^{1}$ \\ ${ }^{1}$ Department of General Surgery, The Second Hospital of Shandong University, Jinan, Shandong 250033; \\ ${ }^{2}$ Department of Histology and Embryology, Shandong University School of Medicine, Jinan, Shandong 250012; \\ Departments of ${ }^{3}$ Health Care For Cadre and ${ }^{4}$ Internal Pediatrics, Qingdao Municipal Hospital, \\ Qingdao, Shandong 266011, P.R. China
}

Received November 11, 2014; Accepted August 17, 2015

DOI: $10.3892 / \mathrm{mmr} .2015 .4297$

\begin{abstract}
Bone morphogenetic proteins (BMPs) belong to the transforming growth factor superfamily and have been implicated in chondrogenesis and neuronal differentiation. In order to examine the function of bone morphogenetic protein 2 (BMP-2) on the differentiation of nitrergic enteric neurons in slow transit constipation (STC), the expression of BMP-2 and neuronal nitric oxide synthase (nNOS) was investigated in the myenteric nerve plexus in STC and control tissues by immunohistochemical assays. The present study demonstrated that BMP-2 and nNOS were expressed in the myenteric nerve plexus and their levels were differentially altered in the STC group and control group. In addition, the effect of BMP-2 on primary myenteric neurons was investigated by measuring the neurite length. The results demonstrated that BMP-2 regulated the differentiation of primary enteric neurons and increased the length of neurites compared with the control group. In addition, the effect of BMP-2 on the expression of nNOS was also investigated in primary enteric neurons and the Smad1 signal transduction pathway by western blot analysis, reverse transcription quantitative polymerase chain reaction and immunofluorescence assay. The results suggested that BMP-2 promoted the expression of nNOS in primary myenteric neurons and induced phosphorylation of Smad1. These data indicate a new role for BMP-2 as an important transcriptional cofactor that regulates the differentiation of nitrergic enteric neurons through the Smad1 pathway. Intervention of BMP-2 may be useful for the treatment of STC.
\end{abstract}

Correspondence to: Professor Daqing Sun, Department of General Surgery, The Second Hospital of Shandong University, 247 BeiYuan Street, Jinan, Shandong 250033, P.R. China

E-mail: sdq15153169151@163.com

${ }^{*}$ Contributed equally

Key words: bone morphogenetic protein 2, neuronal nitric oxide synthase, slow transit constipation, Smad1

\section{Introduction}

Slow transit constipation (STC) is usually considered an intractable colonic motor type of functional constipation. Patients who suffer from the disease tend to be less affected by drug therapy (1) and the most severe cases can require surgery $(2,3)$. Although the pathogenesis of STC remains to be elucidated, numerous theories have attempted to explain its pathogenesis, including a lack of fiber, autonomic neuropathy as well as disorders of the enteric nervous system (ENS) and neuroendocrine system. The ENS derived from neural crest cells is an independent comprehensive nervous network system that consists of ganglionated plexuses, where almost all intrinsic nerve cells reside $(4,5)$. Two ganglionated plexuses are located in the intestine, submucosa and myenteric plexuses, and are known to be involved in all gastrointestinal functions, including motility, secretion, blood flow, mucosal growth and aspects of the local immune system. In pathological situations, the number and type of the enteric neurons may alter. For example, a previous study have demonstrated that the tyrosine hydroxylase phenotype of enteric neurons can be regulated by neuronal activity (6). A marked difference in the expression of several messengers, including vasoactive intestinal peptide, has also been observed in the hypertrophic ileum of patients with Crohn's disease (7). It has been reported that apoptotic phenomena significantly reduced the number of neurons and glial cells in the myenteric plexus in STC patients $(8,9)$. Similar to these findings, the decreased density of neuronal cells was also observed in patients with STC and acquired megacolon (10). A previous study demonstrated that BMP-2 promotes the differentiation of the nitrergic and catecholaminergic enteric neurons in immorto fetal enteric neuronal (IM-FEN) cell lines (11). However, the function of BMP-2 in the differentiation of neuronal nitric oxide synthase (nNOS) in patients with STC has not yet been reported.

Several studies have indicated that bone morphogenetic protein-2 (BMP-2), a member of the transforming growth factor (TGF) $-\beta$ superfamily, is important in the process of regulating the specification, migration, differentiation and aggregation of enteric neurons (12-14). BMPs, for example, 
promote the development of neurotrophin-3 (NT-3)-dependent, TrkC enteric dopaminergic neurons (15). The differentiation of neural ectoderm and the establishment of the neural crest are also regulated by BMPs $(16,17)$. Inhibition of BMP activity using its antagonist Noggin prevents normal migration of enteric neural crest cells in vivo and in vitro (15). Furthermore, neural crest cell migration and ganglion formation in the enteric nervous system were regulated by BMP-2 and the absence of BMP signaling also leads to failure of ganglion formation, with crest cells unable to aggregate into clusters $(18,19)$. Cellular responses to BMP-2 are mediated by the formation of hetero-oligomeric complexes of type I and type II serine/threonine kinase receptors, which are important in the binding and signaling of BMPs (20). Smad1 is an immediate downstream molecule of the BMP-2 receptors (21). Previous studies have revealed that BMP-2 receptors lead to phosphorylation of Smad1/5/8 in a ligand-dependent manner. When phosphorylated, a heteromeric complex is formed by Smad1/5/8 with Smad4, which translocates to the nucleus to control the expression of the target genes.

In the present study, using the primary enteric neurons (from E15 rat embryos), the morphological and neurochemical differentiation effect of BMP-2 was assessed. In addition, the effect of BMP-2 on the expression of nNOS in primary enteric neurons was assessed. Finally, the signal transduction pathways involved were examined, focusing on the Smad1 signaling pathways.

\section{Materials and methods}

Ethical statement. The protocol for this study was approved by the Ethics Committee of Qingdao Municipal Hospital, (Qingdao, China), Shandong Jiaotong Hospital (Jinan, China) and Shandong University, (Jinan, China), and written informed consent was obtained from all study patients.

All animal studies were approved by the Institutional Animal Care and Use Committee of Shandong University. Pregnant Sprague-Dawley rats were obtained from the Animal Experiment Center of Shandong University according to the Institutional Animal Care and Use Committee guidelines and all efforts were made to minimize suffering.

Primary enteric neuron culture. Three Pregnant Sprague-Dawley rats (average weight, $201 \mathrm{~g}$ ) were purchased from the Animal Experiment Center of Shandong University. The rats were kept in a temperature-controlled environment on a 12-h light/dark cycle with free access to food and water. Rats were treated with an overdose of $\mathrm{CO}_{2}$ followed by severing the carotid arteries. All efforts were made to minimize suffering. The embryos (E15; 35-45 per isolation from three pregnant rats) were removed and sacrificed by decapitation. Subsequently, the colon of embryos was removed and finely diced in Hank's balanced salt solution (Sigma-Aldrich, St. Louis, MO, USA). Tissue fragments were collected in $5 \mathrm{ml}$ of medium (DMEM-F12 1:1 medium) and digested at $37^{\circ} \mathrm{C}$ for $15 \mathrm{~min}$ in $0.1 \%$ trypsin (Sigma-Aldrich). The trypsin reaction was terminated by adding $10 \mathrm{ml}$ of medium containing $10 \%$ fetal calf serum and then treated with DNAse I $(0.01 \%$; Sigma-Aldrich) for $10 \mathrm{~min}$ at $37^{\circ} \mathrm{C}$. Following triturating with a $10 \mathrm{ml}$ pipette, cells were centrifuged at $10,620 \mathrm{x} \mathrm{g}$ for
$10 \mathrm{~min}$. Cells were counted and then seeded at a density of $2.4 \times 10^{5}$ cells $\mathrm{cm}^{-2}$ on 24-well plates previously coated for $6 \mathrm{~h}$ with a solution of gelatin (0.5\%; Sigma-Aldrich) in sterile phosphate-buffered saline (PBS). After $24 \mathrm{~h}$, the medium was replaced with serum-free medium (DMEM-F12 1:1 containing $1 \%$ of N-2 supplement; Sigma-Aldrich). Cells were maintained in culture for 7 days. Half of the medium was replaced every other day.

Tissue preparation. All samples were acquired from Qingdao Municipal Hospital and Shandong Jiaotong Hospital. Normal tissues of colon samples were acquired from 20 patients with colon cancer as the control group and 20 patients with STC who received surgery (STC group). Normal samples were collected from areas adjacent to the colon tumor tissue but outside the tumor margins. The control group included 12 males and 8 females, and the STC group comprised 9 males and 11 females. The age of the control group was 67 years and the STC group was 60 years. For histological examination, tissue samples were fixed in $4 \%$ paraformaldehyde in PBS for $24 \mathrm{~h}$ and dehydrated with gradually increasing concentrations of ethanol and embedded in paraffin. A microtome was then used to obtain $5 \mu \mathrm{m}$-thick sections. These prepared slices were used for hematoxylin and eosin (H\&E; Beijing Solarbio Science \& Technology Co Ltd., Shanghai, China) and immunohistochemistry (IHC) staining.

$H \& E$ staining for morphological observation. The paraffin sections were deparaffinized and hydrated in water. The paraffin sections were stained with hematoxylin for $5 \mathrm{~min}$, rinsed several times with distilled water and then stained with eosin for $3 \mathrm{~min}$. Finally, the slides were dehydrated through graded alcohol, made transparent in xylene and mounted with neutral gum. Alterations in basic morphology of the myenteric neuron plexus and smooth muscles were observed under a light microscope (Leica DM3000; Leica Microsystems, Wetzlar, Germany).

IHC. The paraffin-embedded tissue sections were hydrated in xylene (Guangcheng Chemical Reagent Co., Ltd., Tianjin, China) and a graded alcohol series. Antigen retrieval was performed in a water bath at $95^{\circ} \mathrm{C}$ for 20 min with citric acid buffer (Beijing Zhongshan Golden Bridge Biotechnology Co., Ltd., Beijing, China), and endogenous peroxidase activity was blocked with $3 \% \mathrm{H}_{2} \mathrm{O}_{2}$. Next, the tissue sections were incubated with goat serum (Beijing Zhongshan Golden Bridge Biotechnology Co., Ltd.) for $45 \mathrm{~min}$ and stained with rabbit anti-BMP-2 polyclonal antibody (cat. no. SAB1411278; 1:200 dilution; Sigma-Aldrich, Shanghai, China) and mouse anti-nNOS monoclonal antibody (cat. no. sc-5302; 1:200 dilution Santa Cruz Biotechnology Inc., Santa Cruz, CA, USA) at $4^{\circ} \mathrm{C}$ overnight. Following washing with PBS, the tissues were respectively incubated with the biotin-labeled goat anti-rabbit IgG secondary antibody (cat. no. ZDR-5306; 1:800 dilution; Zhongshan Golden Bridge Biological Technology Inc.) and the biotin-labeled goat anti-mouse IgG secondary antibody for $30 \mathrm{~min}$ at $37^{\circ} \mathrm{C}$ (cat. no. ZDR-5307; 1:800 dilution; Zhong Shan Golden Bridge Biological Technology Inc, Beijing, China). Then tissues were stained with 3,3'-diaminobenzidine and hematoxylin (Zhongshan 
Golden Bridge Biological Technology Inc, Beijing, China). The experiment was repeated three times.

Reverse transcription quantitative polymerase chain reaction $(R T-q P C R)$. Primary enteric neurons were cultured in the presence of BMP-2 $(0-20 \mathrm{ng} / \mathrm{ml})$ for $48 \mathrm{~h}$. Total RNA was isolated using TRIzol reagent (Invitrogen Life Technologies, Carlsbad, CA, USA). Total RNA ( $1 \mu \mathrm{g}$ per sample) was reverse-transcribed using the ImProm-II Reverse Transcription System (Promega Corporation, Madison, WI, USA). $\beta$-actin was used as a control. The sequences of the primers used for PCR are shown as follows: nNOS, forward 5'-GGTCGCTTTGAGTACCAGCCT-3' and reverse 5'-GGT CGCTTTGACTCTCTTGG-3'; $\beta$-actin, forward 5'-AAC AGTCCGCCTAGAACACA-3' and reverse 5'-CGTTGACAT CCGTAAAGACC-3'. The product was analyzed on a $1.5 \%$ agarose gel stained with ethidium bromide and the amplified products were visualized by ultraviolet transillumination.

Western blotting. Proteins were obtained from primary enteric neurons treated with BMP-2 (0-20 ng/ml) or its antagonist Noggin $(100 \mathrm{ng} / \mathrm{ml})$ for $48 \mathrm{~h}$. Proteins were resolved on a $10 \%$ SDS-polyacrylamide gel and electroblotted onto a polyvinylidene difluoride membrane (Pall Corporation, Port Washington, NY ,USA). The blots were then incubated with rabbit polyclonal anti-pSmad1 (cat. no. sc-101800, 1:500 dilution; Santa Cruz Biotechnology, Inc., Santa Cruz, CA, USA), mouse monoclonal anti- $\beta$-actin (cat. no. sc-47778 1:2,000 dilution; Santa Cruz Biotechnology, Inc.) or mouse monoclonal anti-nNOS antibody (cat. no. sc-5302 1:500 dilution; Santa Cruz Biotechnology, Inc.). $\beta$-actin was used as a loading control. The membranes were washed and incubated goat anti-rabbit IgG secondary antibody (1:400 dilution; cat. no. sc-45101,Santa Cruz Biotechnology, Inc.) and goat anti-mouse IgG secondary antibody (1:400 dilution cat. no. sc-395763; Santa Cruz Biotechnology Inc.) for $1 \mathrm{~h}$ at $37^{\circ} \mathrm{C}$. The experiment was performed using the SNAP i.d. protein detection system (Millipore, Billerica, MA, USA) according to the manufacturer's instructions and was repeated at least six times. Finally, the signal was visualized following exposure to X-ray film and the relative optical density (OD) ratio was calculated with Image J software 1.4.3.67 (National Institutes of Health, Bethesda, MD, USA) by comparison to $\beta$-actin.

Immunocytochemistry. Primary enteric neurons were treated with the control, BMP-2 (10 ng/ml) or Noggin $(100 \mathrm{ng} / \mathrm{ml})$ for $24 \mathrm{~h}$. Cells were fixed using $4 \%$ paraformaldehyde (30 min) and permeabilized (10 min) with $0.3 \%$ Triton $\mathrm{X}-100$. This was followed by blocking the cells with $3 \%$ normal goat serum (20 min) and overnight incubation with different primary antibodies (anti-nNOS dilution 1:500; anti-pSmad1 dilution 1:500). Secondary detection was performed in conjunction with goat anti-rabbit or anti-mouse $\operatorname{IgG}$ conjugated to fluoresce. Fluorescent photomicrographs were obtained on a Leica microscope (Leica DM1000B).

Morphological assessment of neuronal differentiation. Primary enteric neurons were cultured for 1-4 days in the presence of BMP-2 (0-20 ng/ml). Neuron differentiation was assessed by measurement of neurite length using a phase contrast microscope (Leica DMI3000B).

Immunohistochemical analysis. The expression of BMP-2 and nNOS in the myenteric nerve plexus of STC and control tissues was subjected to microscopic analysis. Following IHC staining, if a cell or tissue was stained from light yellow to brown, it was recorded as positive immunostaining. The areas from STC and its control normal tissue were selected for analysis. The intensity of the staining signal was measured and documented using Image-Pro Plus 6.0 image analysis software (Media Cybernetics, Inc. Silver Spring, MD, USA). The mean densitometry of the digital image (magnification, $\mathrm{x} 400$ ) is designated as representative BMP-2 and $\mathrm{nNOS}$ staining intensity (indicating relative BMP-2 and nNOS expression level). The signal density of tissue areas from five randomly selected fields were counted blindly and subjected to statistical analysis.

Statistical analysis. Statistical analysis was performed using Student's t-test or by one-way analysis of variance with Dunnett's t-test. SPSS 17.0 (SPSS Inc., IBM, Armonk, NY, USA) was used to conduct statistical analysis. Statistical tests were two sided. $\mathrm{P}<0.05$ was considered to indicate a statistically significant difference.

\section{Results}

Expression of BMP-2 and nNOS in myenteric neurons in STC. In order to determine the difference between the control group and the STC group, the morphological changes of myenteric neurons were examined. No apparent morphological difference was identified in the myentric nerve plexus from $\mathrm{H} \& \mathrm{E}$ staining (Fig. 1A and B). The expression of nNOS increased in the STC group and was predominantly located in the myenteric neurons, however, a small number of positive nNOS-expressing neuronal cells were observed in the control group (Fig. 1C and D). In order to determine the association between BMP-2 and the abovementioned variation of nNOS, BMP-2 was detected by IHC staining and it was found that the expression of BMP-2 clearly increased in the STC group compared with the control group (Fig. 1E and F). The mean density of nNOS and BMP-2 was also calculated in STC and control tissues. The results demonstrated that the mean density of nNOS in STC $(0.2257 \pm 0.1095)$ was significantly higher than that of the control tissues $(0.1060 \pm 0.0454, n=20$; $\mathrm{P}<0.05$; Fig. 2A). The results also demonstrated that the mean density of BMP-2 in STC $(0.1993 \pm 0.0847)$ was significantly higher than that of the control tissues $(0.1036 \pm 0.5391, n=20$; $\mathrm{P}<0.05$, Fig. 2B). The results demonstrated that $\mathrm{nNOS}$ and BMP-2 are significantly upregulated in STC tissues compared with control tissues.

BMP-2 induces the differentiation of primary enteric neurons. The effect of BMP-2 on the differentiation of primary enteric neurons (from E15 rat embryos) was examined by measuring neurite length. Neurons were treated with different concentrations of BMP-2 for 1-4 days. The average neurite length was not significantly altered in the $5 \mathrm{ng} / \mathrm{ml} \mathrm{BMP-2}$ group compared with the control group (Fig. 3A and B). However, in 

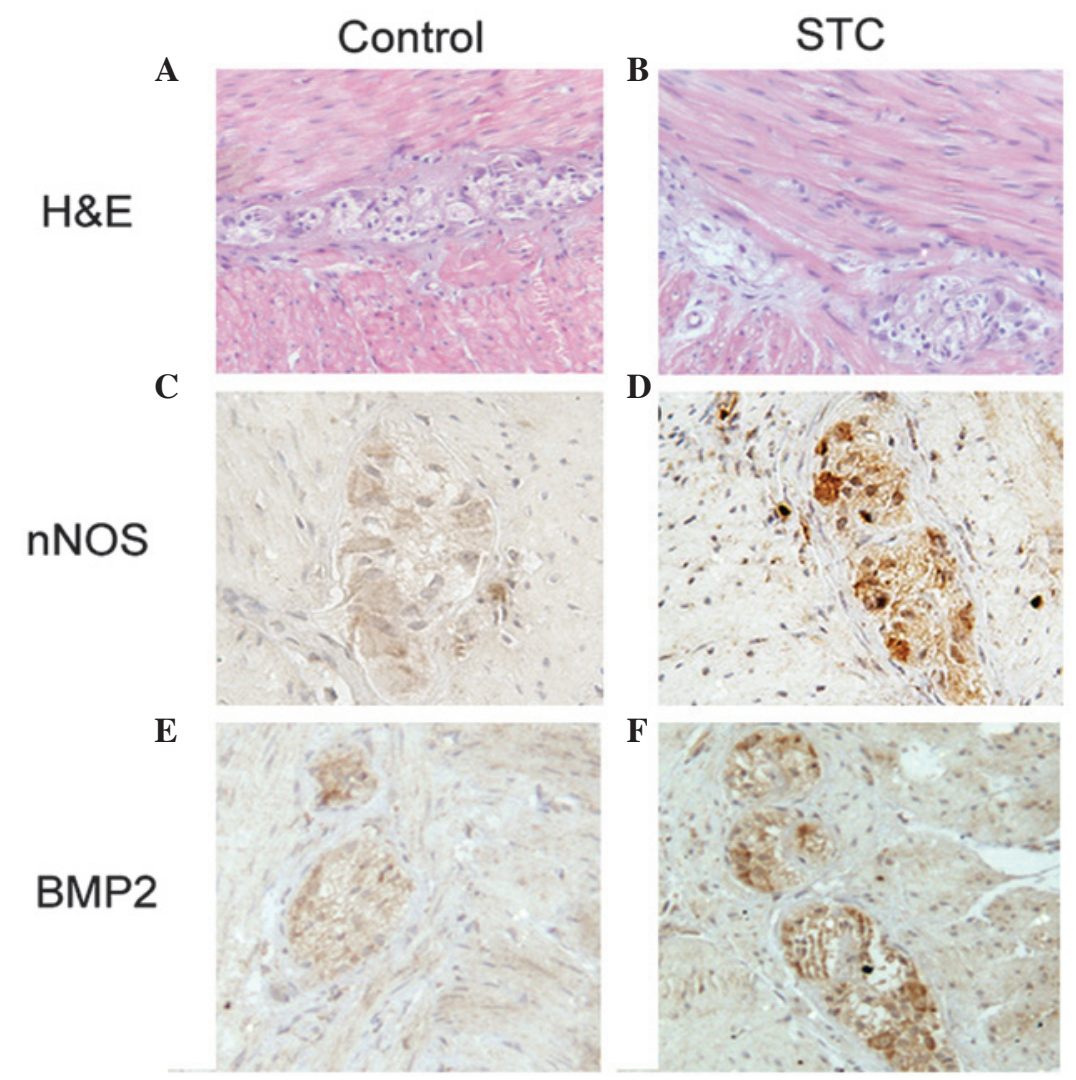

Figure 1. H\&E staining for morphological observation and the expression of nNOS and BMP-2 in the myenteric neurons detected by immunohistochemical staining. (A and B) Colon tissue H\&E staining. No significant difference was identified between the control group and the STC group. (C and D) The left column shows low positive expression of nNOS and the right column shows high positive expression of nNOS. (E and F) The left column shows low positive expression of BMP-2 and the right column shows high positive expression of BMP-2. STC, slow transit constipation; BMP-2, bone morphogenetic protein 2; nNOS, neuronal nitric oxide synthase; H\&E, hematoxylin and eosin. Magnification, $\mathrm{x} 400$.

the $10 \mathrm{ng} / \mathrm{ml}$ (Fig. 3C) or $20 \mathrm{ng} / \mathrm{ml} \mathrm{BMP-2}$ group (Fig. 3D), the average neurite length was significantly increased $(\mathrm{P}<0.05)$. This indicated that in high concentrations, BMP-2 significantly regulated the differentiation of primary enteric neurons and increased the length of neurites compared with the control group (Fig. 3E).

High-concentration BMP-2 markedly increase the expression of $n N O S$. To examine the association between BMP-2 and nNOS in STC, the effect of different concentrations of BMP-2 on nNOS was examined in primary enteric neurons (from E15 rat embryos) in vitro. The primary enteric neurons were treated with BMP-2 $(0-20 \mathrm{ng} / \mathrm{ml})$ for 3 days. The results demonstrated that the induction of nNOS expression by BMP-2 was concentration dependent (Fig. 4). The present study subsequently assessed the effect of BMP-2 on the expression of nNOS by RT-qPCR. The expression of nNOS at the RNA level was almost undetectable in the control or $5 \mathrm{ng} / \mathrm{ml}$ BMP-2 group, however, it significantly increased in the 10 and $20 \mathrm{ng} / \mathrm{ml}$ group ( $\mathrm{P}<0.05$; Fig. 4A and B). Subsequently, the level of nNOS protein was examined by western blot analysis. Similar to the trend of transcripts for nNOS, the protein expression of nNOS was significantly increased in the presence of 10 and $20 \mathrm{ng} / \mathrm{ml}$ BMP-2 compared with the control group and $5 \mathrm{ng} / \mathrm{ml}$ group $(\mathrm{P}<0.05$; Fig. $4 \mathrm{C}$ and $\mathrm{D})$. Furthermore, the effect of BMP-2 or its antagonist Noggin on regulating nNOS expression was determined in primary enteric neurons and it was found that BMP-2 significantly increased the expression of nNOS compared with the control group. However, the effect of BMP-2 on nNOS expression was decreased in the presence of Noggin (Fig. 4E).

BMP-2 induces the phosphorylation and expression of Smadl. Smad1 is an immediate downstream molecule of the BMP-2 receptors (21). To elucidate the signaling pathways involved in BMP-2-induced upregulation of nNOS expression, the effects of BMP-2 stimulation on the phosphorylation of Smad1 protein was examined. The results demonstrated that the phosphorylation of Smad1 protein induced by BMP-2 was concentration dependent. To validate these results, the primary enteric neurons (from E15 rat embryos) were treated with BMP-2 (0-20 ng/ml) for 4 days. The presence of phosphorylated Smad1 was analyzed by western blotting. No significant alterations were observed in $\mathrm{p}$-Smad1 protein content between the control group and $5 \mathrm{ng} / \mathrm{ml}$ BMP-2 group ( $\mathrm{P}>0.05$ ). However, $\mathrm{p}$-Smad1 protein increased markedly in the 10 and $20 \mathrm{ng} / \mathrm{ml}$ BMP-2 group compared with the control and $5 \mathrm{ng} / \mathrm{ml}$ BMP-2 group $(\mathrm{P}<0.05$; Fig. 5A and $\mathrm{C})$. In order to confirm the results, the primary enteric neurons were treated with BMP-2 $(10 \mathrm{ng} / \mathrm{ml})$ and its antagonist Noggin $(100 \mathrm{ng} / \mathrm{ml})$. Western blot analysis demonstrated that phosphorylation of Smad1 protein was significantly increased by BMP-2 and decreased by Noggin compared with the control group ( $\mathrm{P}<0.05$; Fig. 5, B and D). In addition, the percentage of pSmad-positive neurons in the STC 

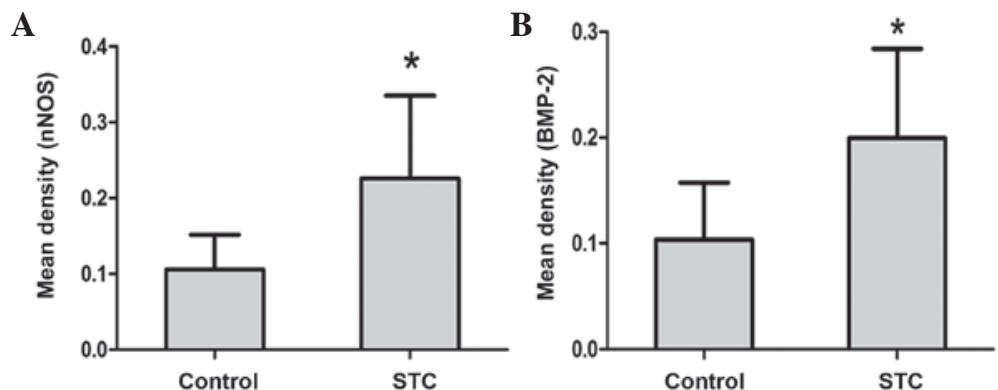

Figure 2. Mean density of nNOS and BMP-2 in STC and control tissue. The mean density of nNOS and BMP-2 from all 20 cases is illustrated in the graph (A) A statistically significant difference was identified between the mean density of nNOS in STC and that in the control tissue. (B) A statistically significant difference was identified between the mean density of BMP-2 in STC and that in the control tissue. "P $<0.05$, compared with the control group. STC, slow transit constipation; BMP-2, bone morphogenetic protein 2; nNOS, neuronal nitric oxide synthase.

A

C
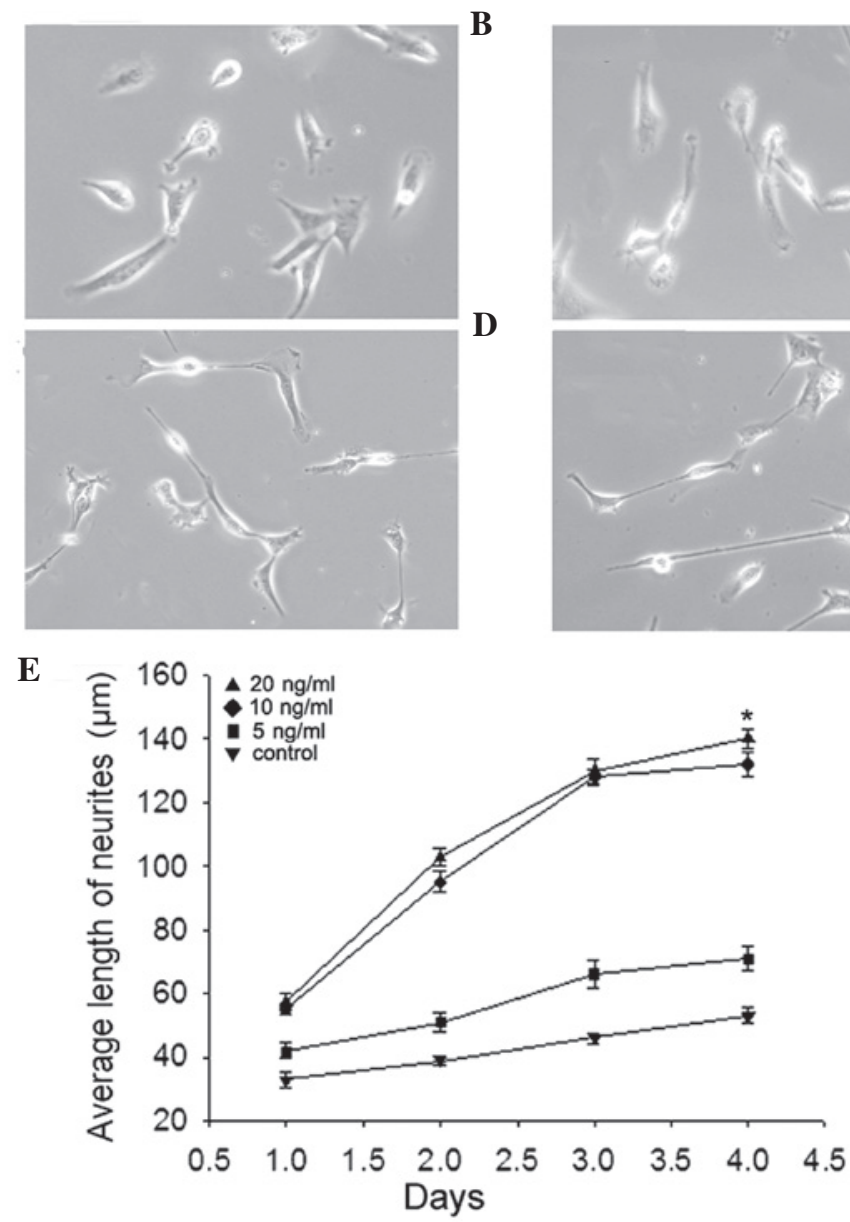

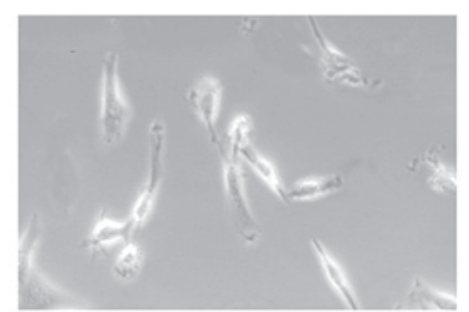

D

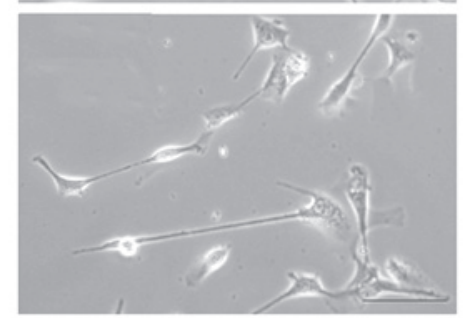

Figure 3. BMP-2 induces differentiation of primary enteric neurons (from E15 rat embryos). (A-D) Phase-contrast photomicrographs of primary enteric neurons cultured in the presence of different doses of BMP-2 (A=0 ng/ml, B=5 ng/ml, C=10 ng/ml and $\mathrm{D}=20 \mathrm{ng} / \mathrm{ml})$. (E) Diagram showing the average length of neurites in primary enteric neurons induced by different concentrations of BMP- 2 . The results are presented as the mean \pm standard deviation $(\mathrm{n}=3$ ). A minimum of 50 neurons were measured in each group. ${ }^{*} \mathrm{P}<0.05$, compared with the control. Scale bar=100 $\mu \mathrm{m}$. BMP-2, bone morphogenetic protein 2 .

group was observed to be more than that in the control group by immunofluorescence (Fig. 5E). These results indicated that the Smad1 pathway is important in BMP-2-induced upregulation of nNOS expression.

\section{Discussion}

The present study demonstrated that BMP-2 regulates the differentiation of nitrergic enteric neurons through the Smad1 pathway in STC. It has been demonstrated that BMPs are essential not only for the development of the neuronal components of the ENS, as previously established, but also for the glial component as well $(15,22,23)$. Using primary enteric neurons, it was demonstrated that BMP-2 regulated the differentiation of nNOS-expressing enteric neurons and may have induced the dysfunction of bowel in STC.

Although the pathogenesis of STC remains to be elucidated, numerous theories have attempted to explain STC, 
A

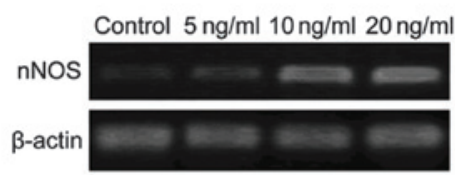

C

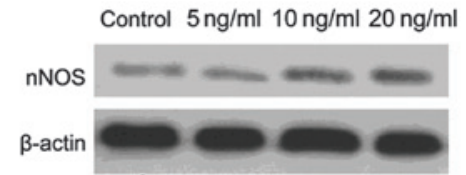

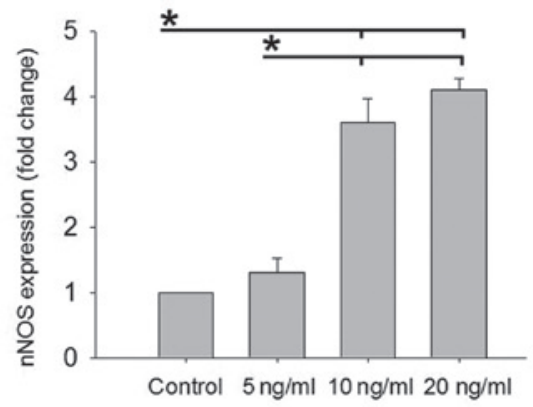

D

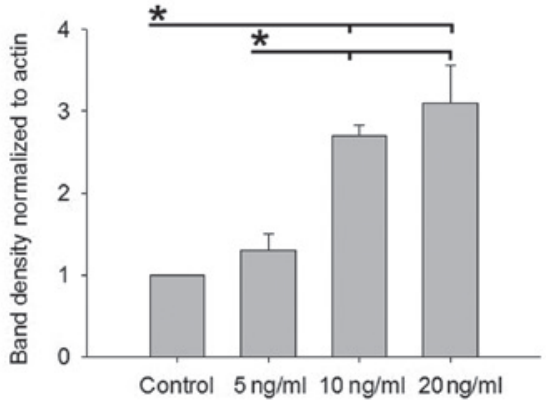

$\mathbf{E}$

nNOS

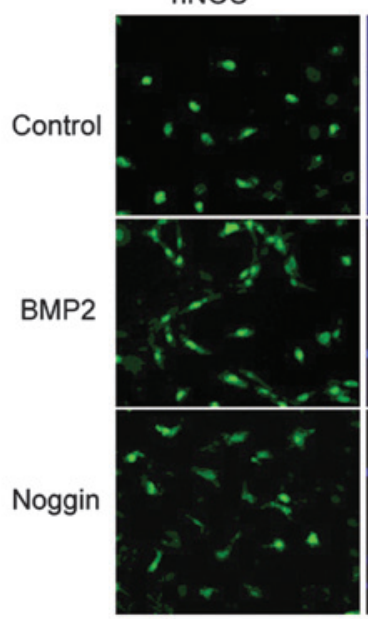

DAPI

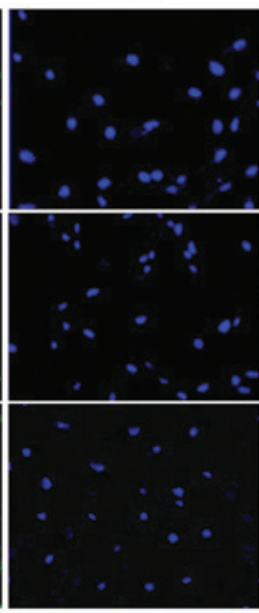

Merge

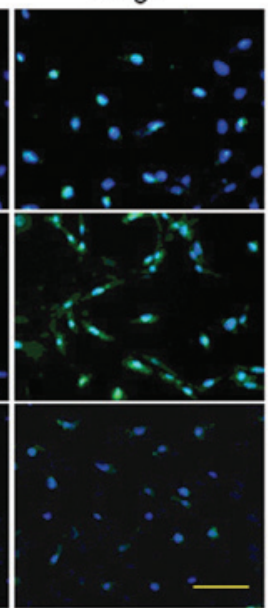

Figure 4. BMP-2 increases the protein expression of nNOS in primary enteric neurons (from E15 rat embryos). (A and B) nNOS mRNA expression level by reverse transcription quantitative polymerase chain reaction in primary enteric neurons treated with $0-20 \mathrm{ng} / \mathrm{ml} \mathrm{BMP}-2$ for $48 \mathrm{~h}$. (C and D) Primary enteric neurons were treated with BMP-2 $(0-20 \mathrm{ng} / \mathrm{ml})$ for 4 days. Representative western blot and densitometry analyses for nNOS protein are shown in primary enteric neurons. $\beta$-actin was used as a loading control. ${ }^{\prime} \mathrm{P}<0.05$. (E) Representative images of primary enteric neurons incubated under control conditions or BMP-2 or with its antagonist Noggin and stained with nNOS. The nuclear stain is shown with DAPI staining. Scale bar=100 $\mu \mathrm{m}$. BMP-2, bone morphogenetic protein 2; nNOS, neuronal nitric oxide synthase; DAPI, 4,6-diamidino-2-phenylindole.

including a lack of fiber, autonomic neuropathy and disorders of the ENS and neuroendocrine system. A previous study indicated that a genetic basis may be present in a subgroup of patients with STC (24). Severely constipated patients have important neuroenteric abnormalities, not confined to ganglion cells and the interstitial cells of Cajal (8). However, it remains unclear whether alterations of enteric neurotransmitters have a role in the pathophysiology of STC. A previous study demonstrated that an excessive production of the neurotransmitter NO in the colonic myenteric plexus of patients with STC contributed to the persistent inhibition of propulsive contractile activity (25). NO is an inorganic molecule with numerous physiological functions and is synthesized from L-arginine by nitric oxide synthase (NOS) (26). Similar to these findings, the increase of NO may be associated with the impaired motility observed in the STC colon $(27,28)$. The expression of nNOS and BMP-2 in the myenteric nerve plexus in patients with STC and control patients was determined using IHC. As a result, it was found that BMP-2 and nNOS were significantly increased in patients with STC compared with patients with colon cancer.

Goldstein et al demonstrated that BMP signaling is necessary for neural crest cell migration and ganglion formation in the ENS and the inhibition of BMP activity leads to 
A

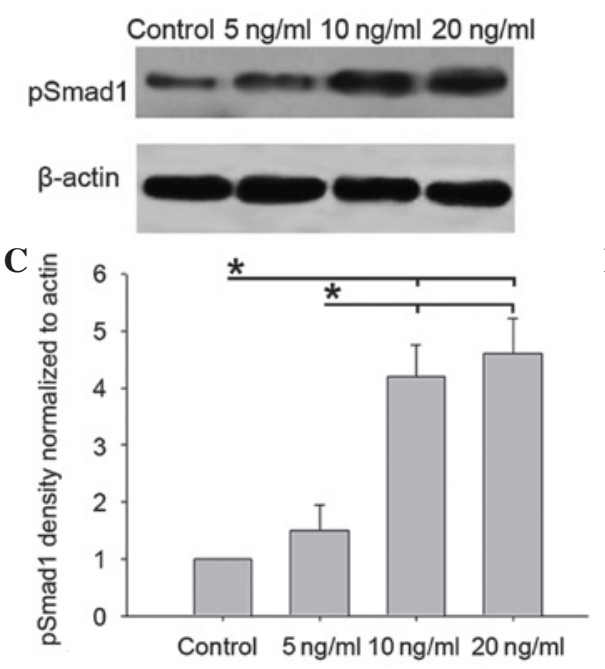

$\mathbf{E}$

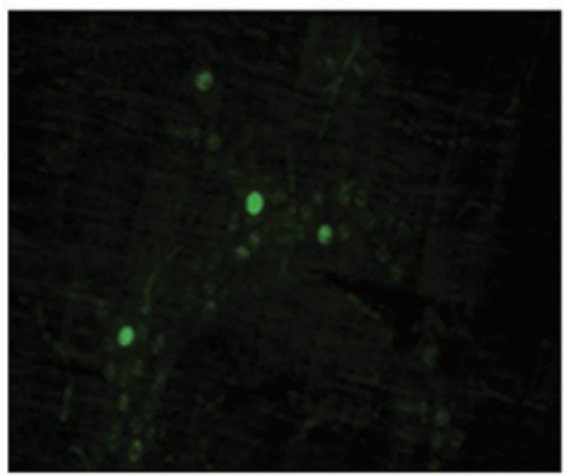

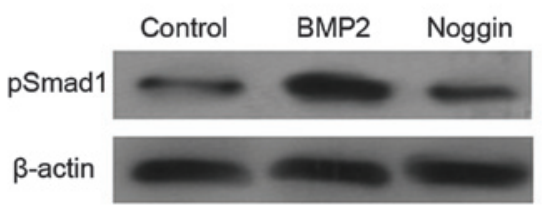

D

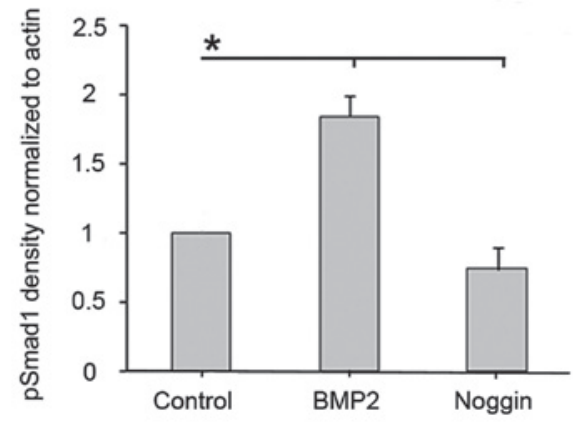

$\mathbf{F}$

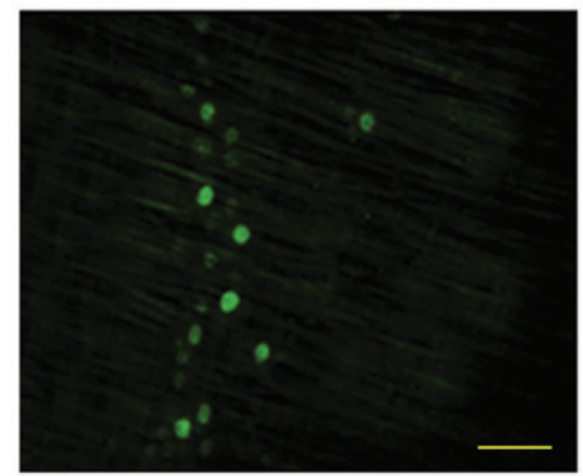

Figure 5. BMP-2 promotes the phosphorylation of Smad1 protein in primary enteric neurons (from E15 rat embryos). (A) Representative western blots of total pSmad1 protein were treated with BMP-2 $(0-20 \mathrm{ng} / \mathrm{ml})$ in primary enteric neurons. (B) Relative mean levels of Smad1 protein are shown in the histogram and $\beta$-actin was used as a loading control. (C) Western blot analysis demonstrated the expression of pSmad1 protein treated with the control, BMP-2 or Noggin. (D) Relative mean levels of Smad1 protein in the BMP-2 group and Noggin group are shown in the histogram. "P<0.05. (E and F) Immunostaining of the enteric neuron plexus showing pSmad1-positive neurons in the STC group and in the control group. Scale bar, $20 \mu \mathrm{m}$. BMP-2, bone morphogenetic protein 2; STC, slow transit constipation.

hypoganglionosis and failure of enteric ganglion formation, with crest cells unable to cluster into aggregates (19). Similar to these findings, in the present study, BMP-2 was found to promote the differentiation of enteric neurons and increase the expression of nNOS in enteric neurons. In addition, the effect of BMP-2 on promoting the expression of nNOS was concentration dependent. Thus, it appears that BMP-2 has a marked effect on the inhibitory subset of enteric neurons. This has therapeutic implications for disease, including STC where the expression of inhibitory neurotransmitters results in dysfunction of intestinal diastole and contraction.

As a result of neurons releasing nNOS, which represent the nitrergic population of enteric neurons, the effect of different concentrations of BMP-2 on the expression of nNOS was next examined in primary enteric neurons. No significant alterations were identified in the protein expression of nNOS and p-Smad1 in the control group and $5 \mathrm{ng} / \mathrm{ml}$ BMP-2 group. However, in the 10 and $20 \mathrm{ng} / \mathrm{ml} \mathrm{BMP-2}$ group nNOS expression was significantly increased. BMP-2 increased the population of nNOS enteric neurons and the expression of nNOS in primary enteric neurons. It has been reported that BMP-6 stimulates macrophages to produce iNOS through Smad signaling pathways and BMP-2 increases nNOS-expressing neurons in IM-FEN cell lines $(11,29)$. These results support the present findings that BMP-2 promotes expression of nNOS in enteric neurons in STC. On the basis of these studies, BMP-2 antagonists may be of potential therapeutic value to reduce the population of nNOS-expressing enteric neurons and ameliorate the dysfunction of bowel in STC disease. Previous studies have demonstrated that BMPs regulate migration and neurite fasciculation within the developing ENS and BMP-2, BMP-4, BMPR-IA (BMP receptor subunit), BMPR-IB, BMPR-II, and the BMP antagonist, Noggin, were all expressed in ENS precursors and intestinal non-neural crest-derived cells at embryonic day 12 (E12) in rats $(15,30)$. Future studies are required to determine whether components of the TGF or BMP signaling pathways can be used as therapeutic targets to prevent or treat dysmotility syndromes, including STC that compromise the function of the ENS. In addition, the effect of BMP-2 on the differentiation of nNOS-expressing enteric neurons and induction of STC has also not been examined in rat models. 
BMPs function mainly through two signal transduction pathways, the Smad-dependent pathway and Smad-independent pathway. Smad signaling is activated and phosphorylated by type I and type II BMP receptor serine/threonine kinases, which form a complex with the common mediator of Smad signaling, Smad4 (16). The Smad complexes subsequently translocate into the nucleus to trigger target gene transcription $(31,32)$. It has been verified that Smad1 signaling is essential for the differentiation of nitrergic enteric neurons induced by BMP-2 in IM-FEN cell lines (12). The present study revealed that BMP-2 can promote the phosphorylation of Smad-1 in primary enteric neurons and the expression of phosphorylated Smad-1 proteins was antagonized by adding Noggin to the cultures of primary enteric neurons. In conclusion, BMP-2 may regulate the expression of nNOS through the Smad1 signaling pathway in enteric neurons of STC.

In conclusion, the results demonstrated that BMP-2 promoted the differentiation of ENS through the Smad1 pathway in STC. In the presence of BMP-2, the expression of nNOS increased significantly in primary enteric neurons. The involvement of BMP-2 in the differentiation of ENS in STC patients is important for a more correct clinical and therapeutic approach.

\section{Acknowledgements}

This study was supported by projects from the Science and Technology Development Program of Shandong Province (grant no. 2012G0021828), the Qingdao Outstanding Health Professional Development Fund, Shandong Provincial Natural Science Foundation (grant no. ZR2014HL016) and the 2015 Qingdao Huimin Project of Science and Technology.

\section{References}

1. Knowles $\mathrm{CH}$ and Martin JE: Slow transit constipation: A model of human gut dysmotility. Review of possible aetiologies. Neurogastroenterol Motil 12: 181-196, 2000.

2. Bassotti G, Chistolini F, Nzepa FS and Morelli A: Colonic propulsive impairment in intractable slow-transit constipation. Arch Surg 138: 1302-1304, 2003.

3. Bassotti G, Roberto GD, Sediari L and Morelli A: Toward a definition of colonic inertia. World J Gastroenterol 10: 2465-2467, 2004.

4. Furness JB, Nguyen TV, Nurgali K and Shimizu Y: The enteric nervous system and its extrinsic connections. In: Textbook of Gastroenterology. Yamada T, Alpers DH, Kalloo AN, Kaplowitz N, Owyang C and Powell DW (eds). Vol 1.5th edition. Blackwell Publishing, Philadelphia, PA, pp15-39, 2003.

5. Furness JB and Costa M: The Enteric Nervous System. Churchill Livingstone, Edinburgh, 1987.

6. Chevalier J, Derkinderen P, Gomes P, Thinard R, Naveilhan P, Vanden Berghe $P$ and Neunlist M: Activity-dependent regulation of tyrosine hydroxylase expression in the enteric nervous system. J Physiol 586: 1963-1975, 2008.

7. Ekblad E, Sjuve R, Arner A and Sundler F: Enteric neuronal plasticity and a reduced number of interstitial cells of Cajal in hypertrophic rat ileum. Gut 42: 836-844, 1998.

8. Bassotti G, Villanacci V, Maurer CA, Fisogni S, Di Fabio F, Cadei M, Morelli A, Panagiotis T, Cathomas G and Salerni B: The role of glial cells and apoptosis of enteric neurones in the neuropathology of intractable slow transit constipation. Gut 55: 41-46, 2006.

9. Bassotti G and Villanacci V: Slow transit constipation: A functional disorder becomes an enteric neuropathy. World J Gastroenterol 12: 4609-4613, 2006.

10. Lee JI, Park H, Kamm MA and Talbot IC: Decreased density of interstitial cells of Cajal and neuronal cells in patients with slow-transit constipation and acquired megacolon.J Gastroenterol Hepatol 20: 1292-1298, 2005.
11. Anitha M, Shahnavaz N, Qayed E, Joseph I, Gossrau G, Mwangi S, Sitaraman SV, Greene JG and Srinivasan S: BMP2 promotes differentiation of nitrergic and catecholaminergic enteric neurons through a Smad1-dependent pathway. Am J Physiol Gastrointest Liver Physiol 298: G375-G383, 2010.

12. Katagiri T, Akiyama S, Namiki M, Komaki M, Yamaguchi A, Rosen V, Wozney JM, Fujisawa-Sehara A and Suda T: Bone morphogenetic protein-2 inhibits terminal differentiation of myogenic cells by suppressing the transcriptional activity of MyoD and myogenin. Exp Cell Res 230: 342-351, 1997.

13. Aoyama K, Yamane A, Suga T, Suzuki E, Fukui T and Nakamura Y: Bone morphogenetic protein-2 functions as a negative regulator in the differentiation of myoblasts, but not as an inducer for the formations of cartilage and bone in mouse embryonic tongue. BMC Dev Biol 11: 44, 2011.

14. Liu A and Niswander LA: Bone morphogenetic protein signalling and vertebrate nervous system development. Nat Rev Neurosci 6: 945-954, 2005.

15. Chalazonitis A, D'Autréaux F, Guha U, Pham TD,Faure C, Chen JJ, Roman D, Kan L, Rothman TP, Kessler JA and Gershon MD: Bone morphogenetic protein-2 and -4 limit the number of enteric neurons but promote development of a TrkC-expressing neurotrophin-3-dependent subset. J Neurosci 24: 4266-4282, 2004.

16. Chen D, Zhao M and Mundy GR: Bone morphogenetic proteins. Growth Factors 22: 233-241, 2004.

17. Kishigami S and Mishina Y: BMP signaling and early embryonic patterning. Cytokine Growth Factor Rev 16: 265-278, 2005.

18. Roberts DJ, Johnson RL, Burke AC, Nelson CE, Morgan BA and Tabin C: Sonic hedgehog is an endodermal signal inducing Bmp-4 and Hox genes during induction and regionalization of the chick hindgut. Development 121: 3163-3174, 1995.

19. Goldstein AM, Brewer KC, Doyle AM, Nagy N and Roberts DJ: BMP signaling is necessary for neural crest cell migration and ganglion formation in the enteric nervous system. Mech Dev 122: 821-833, 2005.

20. Kawabata M, Imamura T and Miyazono K: Signal transduction by bone morphogenetic proteins. Cytokine Growth Factor Rev 9: 49-61, 1998.

21. Hoodless PA, Haerry T, Abdollah S, Stapleton M, O'Connor MB, Attisano L and Wrana JL: MADR1, a MAD-related protein that functions in BMP2 signaling pathways. Cell 85: 489-500, 1996.

22. Chalazonitis A, Pham TD, Li Z, Roman D, Guha U, Gomes W, Kan L, Kessler JA and Gershon MD: Bone morphogenetic protein regulation of enteric neuronal phenotypic diversity: Relationship to timing of cell cycle exit. J Comp Neurol 509: 474-492, 2008.

23. Chalazonitis A, D'Autreaux F, Pham TD, Kessler JA and Gershon MD: Bone morphogenetic proteins regulate enteric gliogenesis by modulating ErbB3 signaling. Dev Biol 350: 64-79, 2011.

24. Rossi E, Villanacci V, Fisogni S, Morelli A, Salerni B, Grigolato P and Bassotti G: Chromosomal study of enteric glial cells and neurons by fluorescence in situ hybridization in slow transit constipation. Neurogastroenterol Motil 19: 578-584, 2007.

25. Tomita R, Fujisaki S, Ikeda T and Fukuzawa M: Role of nitric oxide in the colon of patients with slow-transit constipation. Dis Colon Rectum 45: 593-600, 2002.

26. Moncada S, Palmer RM and Higgs EA: Nitric oxide: Physiology, pathophysiology, and pharmacology. Pharmacol Rev 43: 109-142, 1991.

27. Saur D, Paehge H, Schusdziarra V and Allescher HD: Distinct expression of splice variants of neuronal nitric oxide synthase in the human gastrointestinal tract. Gastroenterology 118: 849-858, 2000.

28. Porter AJ, Wattchow DA, Hunter A and Costa M: Abnormalities of nerve fibers in the circular muscle of patients with slow transit constipation. Int J Colorectal Dis 13: 208-216, 1998.

29. Kwon SJ, Lee GT, Lee JH, Kim WJ and Kim IY: Bone morphogenetic protein- 6 induces the expression of inducible nitric oxide synthase in macrophages. Immunology 128 (Suppl 1): e758-e765, 2009.

30. Fu M, Vohra BP, Wind D and Heuckeroth RO: BMP signaling regulates murine enteric nervous system precursor migration, neurite fasciculation, and patterning via altered Ncam1 polysialic acid addition. Dev Biol 299: 137-150, 2006.

31. Piek E, Heldin CH and Ten Dijke P: Specificity, diversity, and regulation in TGF-beta superfamily signaling. FASEB J 13: 2105-2124, 1999.

32. Heldin $\mathrm{CH}$, Miyazono K and Ten Dijke P: TGF-beta signalling from cell membrane to nucleus through SMAD proteins. Nature 390: 465-471, 1997. 